Significant negative correlation between resilience and emotional exhaustion $(r=-0.36, p=0.005)$ and positive correlation between resilience and personal achievement $(r=0.36, p=0.005)$.

No significant difference in coping strategies across different levels of resilience, only one ('keep professional boundaries') distinguished between higher and lower resilience levels.

Conclusion Paediatric and Neonatal ICU staff show concerning levels of psychological trauma. Greater resilience is associated with lower levels of PTSD symptomatology and some measures of burnout, indicating a better psychological state.

More research is required to investigate the role of different coping strategies, including techniques, which can influence resilience, and how these may be promoted in staff working in this environment to facilitate staff wellbeing and retention.

\section{G50 ADMISSION OF EX-PREMATURE BABIES TO PICU IN THEIR FIRST TWO YEARS OF LIFE}

doi:10.1136/archdischild-2013-304107.062

HL Yates. Neonatal Services, Leeds Teaching Hospital Trust, Leeds, UK

Aim To asses the proportion of ex-premature babies who go on to be admitted to PICU (Paediatric Intensive Care) in their first 2 years of life, and the way in which their gestation modifies treatment requirements.

Method PICANet (National PICU database) admission data were retrieved for all PICU admissions in England and Wales who were under 2 years old between 01/01/2007 and 31/12/2010 with gestation less than 36 weeks. 2007-2008 gestation specific live-birth and neonatal mortality rates for England and Wales have been published by the Office of National Statistics. Data was analysed in Microsoft Excel,with Chi-squared and Kruskal-Wallis tests, with statistical significance at $5 \%$ level.

Result Birth gestation was recorded for 70\% (11559/16680) of the studied PICU admissions.

$3.8 \%$ of babies born in England and Wales in 2007-2008 were born at less than 36 weeks gestation. $3.2 \%$ of these babies went on to be admitted to PICU before their second birthday, comprising $12.4 \%$ of all PICU admissions in the under $2 \mathrm{~s}$.

Late pre-term babies account for a higher number of PICU admissions overall. (See Figure 1). However, the admission rate, when expressed as the percentage of those alive at day 28 , is higher among extreme pre-term babies. (See Figure 2)

Statistically significant differences between gestational cohorts were seen in the median length of stay (see Figure 3) and median duration of ventilation. No difference in PICU associated mortality was seen.

Conclusion A higher percentage of extreme preterm babies than late preterm babies are admitted to PICU in their first 2 years of life. They require longer stays and longer durations of ventilation.

The rising preterm birth rate coupled with increasing survival of extreme preterm babies will have a significant impact on PICU demand.

\section{G51 ADMISSIONS TO PICU IN ENGLAND AND WALES WITH REFRACTORY CONVULSIVE STATUS EPILEPTICUS: A TWO- YEAR NATIONAL EPIDEMIOLOGICAL STUDY}

doi:10.1136/archdischild-2013-304107.063

${ }^{1,2} \mathrm{IJ}$ Tully, ${ }^{3} \mathrm{C}$ Lamming, ${ }^{3} \mathrm{E}$ Draper, ${ }^{4} \mathrm{D}$ Mattison, ${ }^{1} \mathrm{C}$ Thomas, ${ }^{4} \mathrm{~T}$ Martland, ${ }^{1} \mathrm{RE}$ Appleton. ${ }^{1}$ Neurology, Alder Hey Children's Hospital, Liverpool, UK; '²Paediatrics, Caboolture Public Hospital, Caboolture, Australia; ${ }^{3}$ Health Sciences, Leicester University, Leicester, UK; ${ }^{4}$ Neurology, Royal Manchester Children's Hospital, Manchester, UK

Aims To obtain epidemiological data on the prevalence, aetiology, management and outcome of refractory convulsive status epilepticus (RCSE).

Methods Data on children admitted with RCSE to eight paediatric intensive care units (PICUs) were collected retrospectively using a standard proforma designed and co-ordinated by PICAnet. Data were collected between 31/12/2007 and 31/12/2009.

Results Data were collected on 245 (male, 179) patients aged $<1$ month to 16.5 years (mean 3.8, median 2.8 years). Causes included acute symptomatic (12.4\%), remote symptomatic $(18.4 \%)$, epilepsy-related $(22.4 \%)$, progressive encephalopathy $(5.3 \%)$ and febrile (14.7\%); no cause was identified in $23.7 \%$.

Thirty nine patients received treatment (midazolam > diazepam) at home and 73 received treatment from paramedics (diazepam $>$ midazolam).

In the Emergency Department (ED), 219 patients (89\%) received at least one benzodiazepine dose, $197(80.4 \%)$ received phenytoin and $23(9.3 \%)$ received phenobarbital. Subsequent anticonvulsants

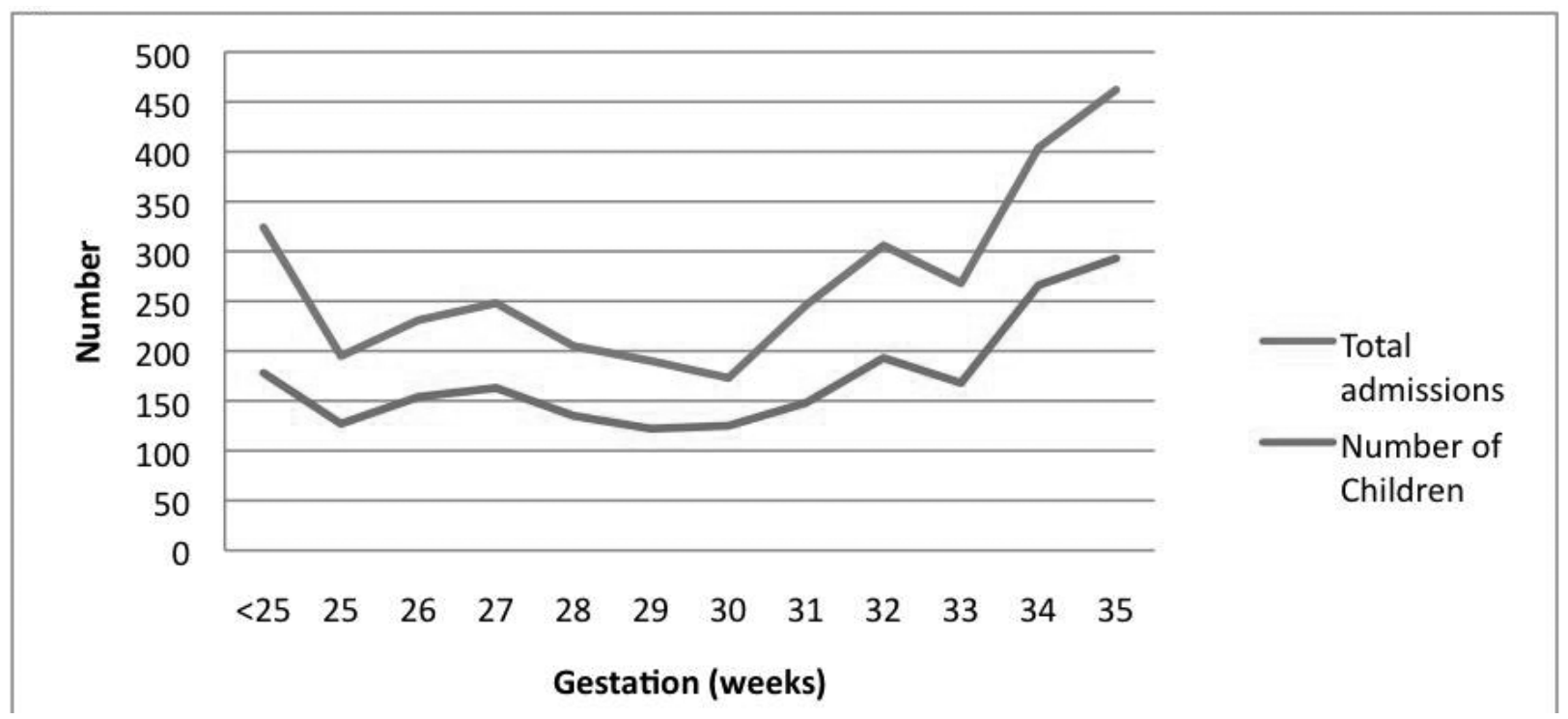

Abstract G50 Figure 1 Number of ex-premature babies admitted to PICU in the first 2 years of life by gestation. 